\title{
Relation between testosterone levels and body composition, physical functioning and selected biochemical parameters in adult males
}

\author{
Milan Sedliak', Michal Kralik', Jan Cvecka', Gabriel Buzgo', Matus Putala', Barbara Ukropcova', , Jozef \\ Ukropec $^{2}$, Zdenko Kilinger ${ }^{3}$, Juraj Payer ${ }^{3}$, Boris Kollarik ${ }^{4}$, Peter Bujdak ${ }^{5}$, Truls Raastad ${ }^{6}$ \\ ${ }^{1}$ Department of Biological and Medical Sciences, Faculty of Physical Education and Sport, Comenius University \\ in Bratislava, Slovak Republic \\ ${ }^{2}$ Department of Metabolic Disease Research, Institute of Experimental Endocrinology, Biomedical Research \\ Center, Slovak Academy of Sciences, Bratislava, Slovak Republic \\ ${ }^{3} 5^{\text {th }}$ Department of Internal Medicine, Faculty of Medicine, Comenius University in Bratislava, University Hospital, \\ Bratislava, Slovak Republic \\ ${ }^{4}$ Department of Urology, University Hospital, Bratislava, Slovak Republic \\ ${ }^{5}$ Deparment of Urology, Faculty of Medicine, Comenius University in Bratislava, University Hospital, Bratislava, \\ Slovak Republic \\ ${ }^{6}$ Department of Physical Performance, Norwegian School of Sport Sciences, Oslo, Norway \\ ${ }^{7}$ Institute of Pathophysiology, Faculty of Medicine, Comenius University in Bratislava, Bratislava, Slovak Republic
}

Introduction: The objective of the study was to examine the relationship between the values of selected parameters of physical function, body composition, body mass index (BMI) and biochemical markers of metabolic health with the total testosterone (TT) levels in adult males. We aimed to analyse the correlation between these values and variations in the TT levels. Methods: A total of 17 subjects (age $=50.2 \pm 8.1$ years, TT $=11.4 \pm 3.8 \mathrm{nmol} / \mathrm{l}$ ) were included in the study. Subjects were tested on physical function (1RM on leg press, bench-press, handgrip, $\mathrm{VO}_{2 \max }$ ), body composition (DXA), biochemical parameters (morning fasting blood samples). Results: TT was inversely correlated with abdominal circumference $(A C)(p<0.01)$ and with overall body fat, measured in $\mathrm{kg}(\mathrm{p}<0.01)$. On a biochemical level, significant correlations were found between TT and insulin $(p<0.01)$, and TT and homeostasis model assessment of insulin resistance (HOMA-IR) $(p<0.01)$. Physical function, muscle strength or lean mass were not significantly correlated with TT. Conclusion: The main finding of this study was that testosterone levels had a strong inverse correlation with abdominal circumference and total body fat mass. On metabolic level, strong inverse correlation was also found between TT with insulin and TT with HOMA-IR. However, we did not find statistically significant correlation between total testosterone levels and lean mass, muscle strength or physical function in middle aged males.

Key words: body composition, obesity, testosterone. 


\section{Introduction}

The Endocrine Society defines a clinical syndrome that results from failure of the testis to produce physiological levels of testosterone (androgen deficiency) as male hypogonadism (1). Besides the total serum testosterone levels below a physiological range (total testosterone levels $<6.9 \mathrm{nmol} / \mathrm{l}$ ), symptoms include decline in lean mass, muscle strength, increased adiposity, decreased libido and erectile dysfunction, depressed mood, decreased energy or vitality, increased fatigue, and osteoporotic fractures. The symptoms mentioned above may affect males already in their late thirties. Some studies of hypogonadal men showed that low testosterone level is also highly related to insulin resistance, visceral obesity and metabolic syndrome (2). Normalization of testosterone level should be the primary treatment in men, along with caloric restriction and physical exercise. Muscle strength determines mobility and physical functioning and thus, also does affect the quality of life. Some studies suggest that aged men could benefit from testosterone replacement regarding muscle mass preservation The direct correlation of the therapy, however, and its direct impact on strength and functional status is unproven. Testosterone treatment may provide modest improvements in lean mass among both frail and non-frail older men, but current evidence on the testosterone effect on muscle strength is conflicting and the effect on physical function is weak (3). However, other authors (4) found, that in older men, low circulating testosterone is correlated with low muscle strength, with high adiposity, with insulin resistance and with poor cognitive performance. Data from Massachusetts Male Aging Study (MMAS) found that testosterone concentration up to a critical level were positively correlated with muscle strength.

Chronic lower levels of testosterone dramatically increase the risk of many other diseases as cardiovascular disease, sexual dysfunction, aortic atherosclerosis, Alzheimer's disease and others (5). The relationship between total testosterone levels (TT) and high density lipoproteins (HDL) is confounded by the fact that both HDL and TT are inversely related to body mass index (BMI). In fact, epidemiological analyses have found that HDL levels are positively linked to testosterone levels in middle-aged men. Data from the Massachusetts Male Aging Study (MMAS) have demonstrated that there is a strong, positive relationship between HDL and testosterone in men with cardiovascular disease (low total or free testosterone correlates with low HDL-cholesterol) (6).

On a metabolic level, in obese men, hypogonadism can further worsen the metabolic profile and increase abdominal fat (7). Chronic effects of testosterone deficiency have effects on leg adipose tissue acyl-CoA synthetase activity which may relate to greater lower body fatty acid storage (8). These morphological features are linked to metabolic dysfunction, and testosterone deficiency is associated with energy imbalance, impaired glucose control, reduced insulin sensitivity and dyslipidaemia (9). Data suggest that abdominal circumference alone could be used as an anthropometric parameter to help simplify the identification of men with low serum total testosterone levels. The main goal of the study based on previous studies was to examine the relationship between the values of selected parameters of physical function, body composition, body mass index (BMI) and biochemical markers of metabolic health with the total testosterone (TT) levels in adult males. We aimed to analyse the correlation between these values and variations in the TT levels.

\section{Methods}

\section{Subjects}

Subjects were recruited from urological units at the Department of Urology, University Hospital-Petrzalka, Bratislava, Slovakia and the Department of Urology, Faculty of Medicine, Comenius University, Bratislava, Slovakia. The inclusion criteria for participation in the study of the patient population included age 40-60 years old, diagnosed with hypogonadism on testosterone replacement therapy or newly diagnosed patients. There was also a group of healthy aged matched males serving as a control group. For a purpose of this manuscript, all the groups were merged together and are presented as a one group in order of higher number of subjects for correlation analyses, which are the main focus of the present study. The exclusion criteria included regular strength training, conditions which are medical contraindications prostate cancer or abnormal serum PSA levels without adverse histological examination. In addition to written information, eligible subjects are verbally informed about the study by their responsible urologist and the study officials before start of the tests.

Seventeen adult males (50.19 \pm 8.07 years old, $92.4 \pm 12.83 \mathrm{~kg}$ ) participated in the study. Complete demographic and hormonal data of the group are presented (tab. 1).

The study was approved by Ethics Committee of the Derer's Memorial Hospital in Bratislava, Slovakia and all subjects provided and signed written informed consent.

\section{Design}

To secure validity of the physical tests, all subjects underwent a familiarization session 5-7 days prior to the actual assessments. During the time between familiarization and actual testing, morning blood samples were collected and body composition measurements took place also in the morning hours.

\section{Body composition}

Body composition was measured by Dual-energy X-ray Absorptiometry (DXA) using Hologic fan-beam bone densitometer Discovery QDR series. Lean mass (LM), fat mass (FM) and total body mass (BM) were measured and are presented. The height was measured in meters by stadiometer and abdominal circumference in centimetres (cm) by stretch-resistant tape that provides a constant $100 \mathrm{~g}$ tension. The landmark of the average point between the $10^{\text {th }}$ rib and the iliac crest at the axillary line. The body mass index (BMI) was afterwards calculated from the DXA scan data.

\section{Physical function}

Muscle strength of lower extremities was measured by predicted dynamic leg press 1RM from multiple repetition maximum testing (10) on seated leg press machine (Technogym, Italy). For upper extremities, 
Tab. 1. Demographic and biochemical data

\begin{tabular}{|c|c|}
\hline$N=17$ & average \pm SD \\
\hline Age (year) & $50.2 \pm 8.1$ \\
\hline Total body weight (kg) & $92.37 \pm 19.49$ \\
\hline Lean mass (kg) & $62.55 \pm 7.46$ \\
\hline Overall body fat mass (kg) & $29.82 \pm 12.71$ \\
\hline Overall body fat mass (\%) & $31.34 \pm 5.55$ \\
\hline BMI $\left(\mathrm{kg} \cdot \mathrm{m}^{-2}\right)$ & $30.04 \pm 6.50$ \\
\hline Abdominal circumference $(\mathrm{cm})$ & $104.50 \pm 9.96$ \\
\hline Total testosterone $(\mathrm{nmol} / \mathrm{l})$ & $11.45 \pm 3.81(6.9-26.1)$ \\
\hline SHBG (nmol/l) & $30.00 \pm 11.62(14.5-94.6)$ \\
\hline Total cortisol (nmol/l) & $491.87 \pm 87.48(118.6-618)$ \\
\hline Total cholesterol (mmol/l) & $5.34 \pm 1.33(3.2-5)$ \\
\hline LDL-cholesterol (mmol/l) & $3.68 \pm 1.07(1-3)$ \\
\hline HDL-cholesterol (mmol/l) & $1.19 \pm 0.20(1-2)$ \\
\hline HOMA-IR & $3.96 \pm 2.99(0.4-2.0)$ \\
\hline Glucose $(\mathrm{mmol} / \mathrm{l})$ & $5.44 \pm 1.33(3.3-5.6)$ \\
\hline Insulin (mIU/l) & $15.82 \pm 10.35(3-25)$ \\
\hline $\begin{array}{l}\text { Predicted Legress } 1 \mathrm{RM} \text { relative to lean } \\
\text { mass (kg) }\end{array}$ & $2.49 \pm 0.44$ \\
\hline Handgrip average (kg) & $50.26 \pm 7.68$ \\
\hline${ }^{*} \mathrm{VO}_{2 \max }\left(\mathrm{mlO}{ }_{2} \cdot \mathrm{kg}\right.$ BW-1.min-1) & $33.88 \pm 1.12$ \\
\hline
\end{tabular}

Data are presented as means of the group with standard deviations. Biochemical parameters were analysed from fasting morning plasma. Reference values for a local male population are in parentheses.

BMI - Body Mass Index, SHBG - Sex Hormone Binding Globulin, LDL - Low Density Lipoprotein, HDL - High Density Lipoprotein, HOMA-IR-The Homeostatic Model Assessment - Insulin Resistance, *indirect assessment handgrip strength was measured by best of the 3 trials with 60 second rest period after warming up with 3 trials (approximately 50 and twice $80 \%$ of maximal voluntary contraction) with the resting period of 20 seconds using digital hand dynamometer (Camry, USA).

Cardio-respiratory fitness was measured by The Single Stage Treadmill (Woodway Pro, USA) Walking Test, which is a submaximal aerobic fitness test that estimates $\mathrm{VO}_{2 \max }$. It is suitable for low risk, apparently healthy, non-athletic adults 20-59 years of age. The walking pace required throughout the test also makes it appropriate for participants who experience problems such as knee pain when exercising at a jogging pace. The test can be administered to moderate sized groups of participants with low to moderate fitness levels and requires only a treadmill and a $\mathrm{HR}$ monitor $\mathrm{VO}_{2 \max }$ was estimated from heart rate results of the walking test using protocols calculation (11).

\section{Biochemical parameters}

Fasting morning venous blood samples were collected between 8:00 AM to 10:00 AM into closed system collection tubes containing beads coated with a clotting activator and polyacryl ester-gel (Sarstedt AG \& Co, Germany). The samples were centrifuged at 2000 rpm for 10 minutes at $4^{\circ} \mathrm{C}$ no later than 60 minutes after the blood draw to generate a serum and immediately transported to the accredited medical laboratory for further analyses. The parameters analysed from serum were glucose, total cholesterol, LDL-cholesterol, HDL-cholesterol, total

Fig. 1. Relationship between total testosterone levels and abdominal circumference $(r=-0.639, p<0.01$

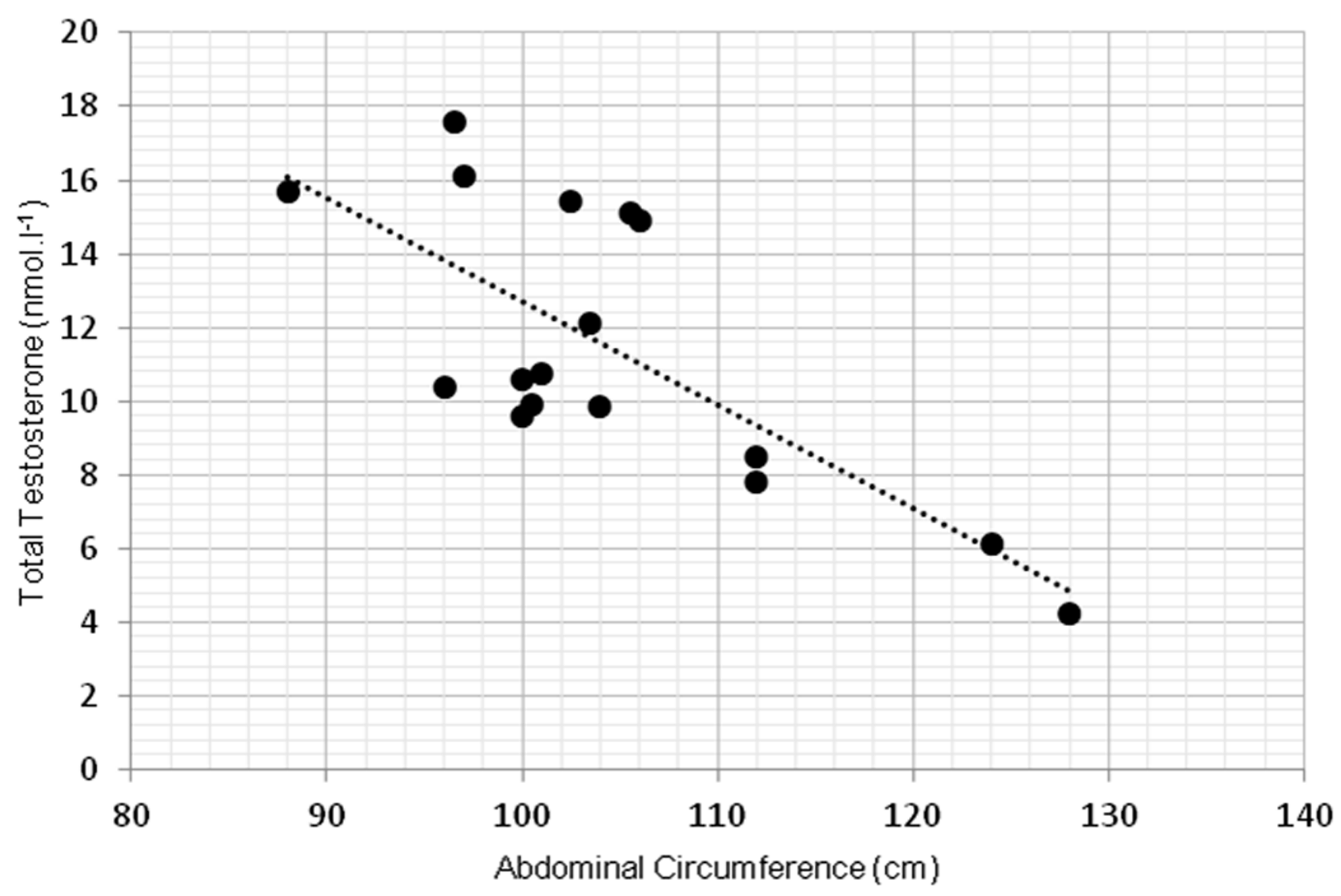


Fig. 2. Relationship between total testosteronelevels and overall body $f a t(r=-0.762, p<0.01)$

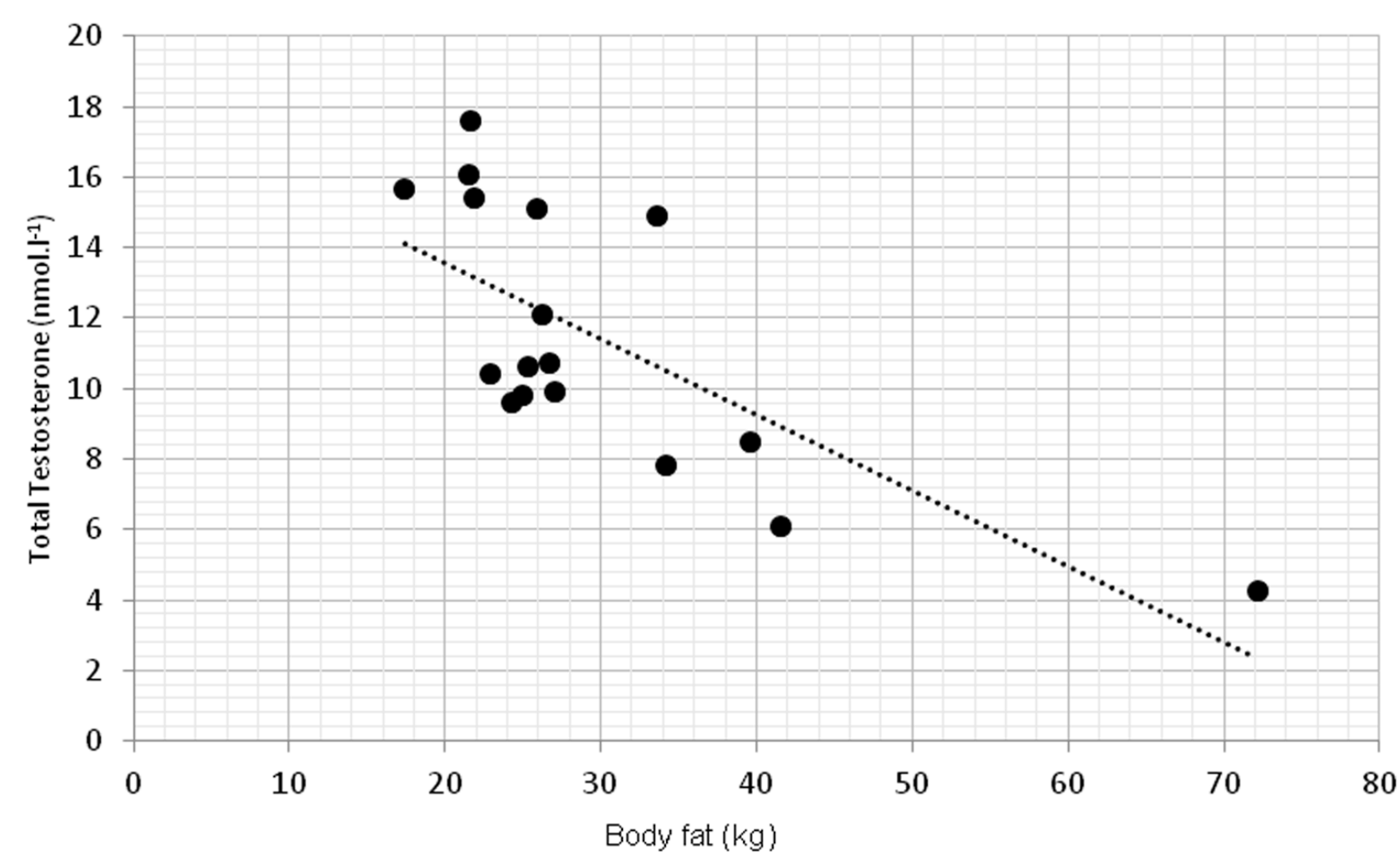

Fig. 3. Relationship between total testosterone levels and insulin $(r=-0.674, p<0.01)$

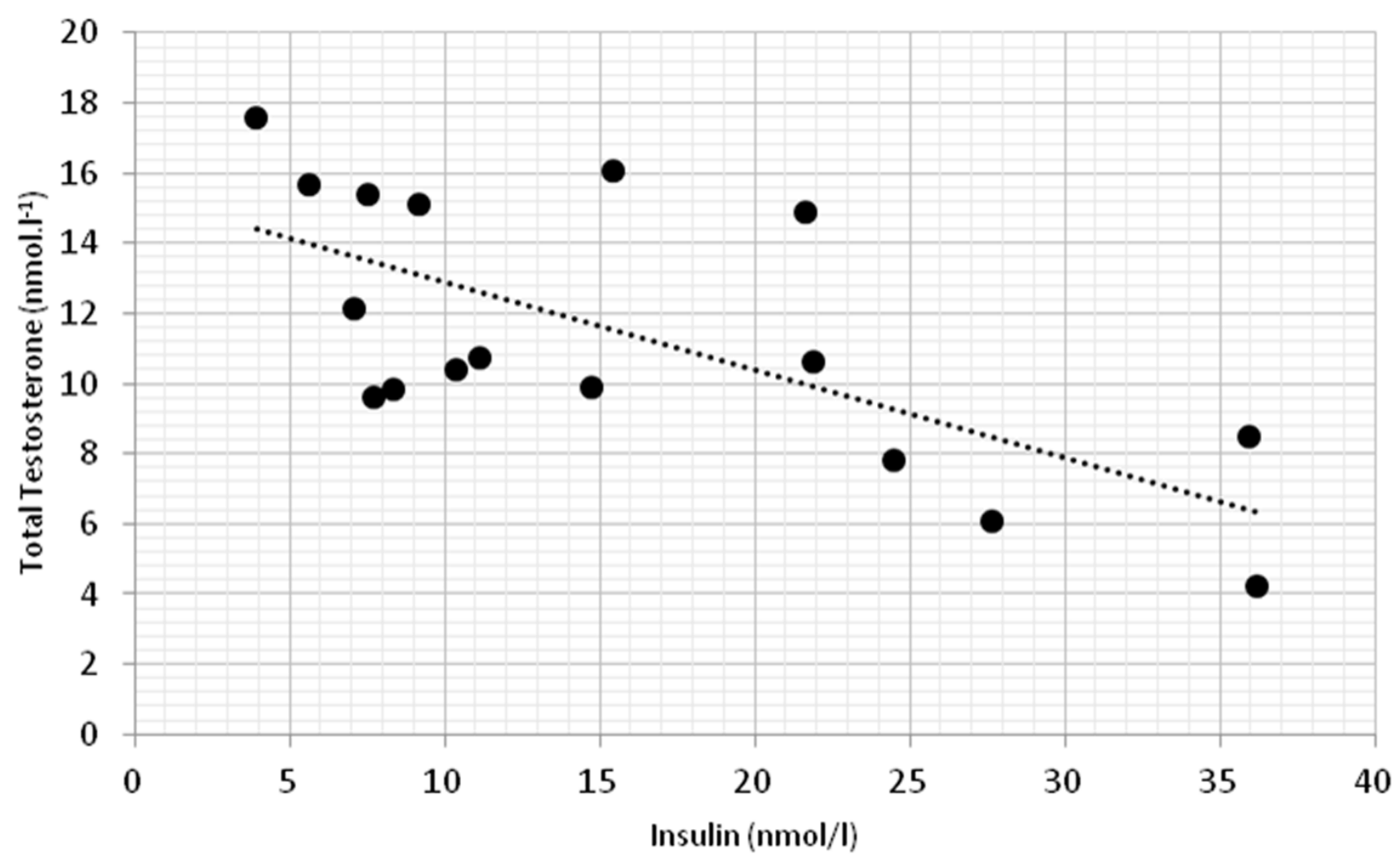


Fig. 4. Relationship between total testosterone levels and HOMA-IR $(r=-0.623, p<0.01)$

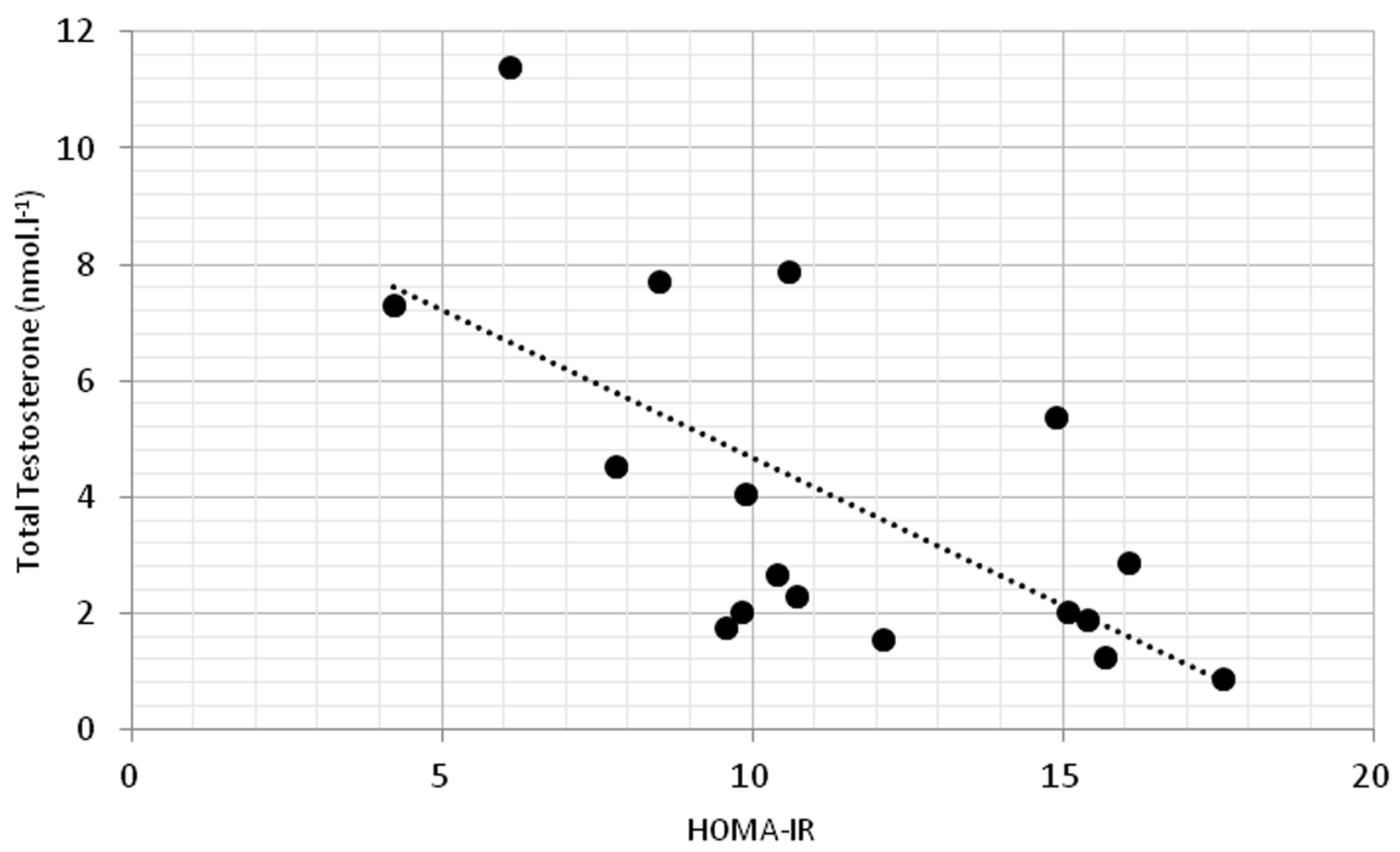

testosterone (TT), SHBG, insulin, homeostasis model assessment of insulin resistance (HOMA-IR) and cortisol.

\section{Results}

Due to limited number of subjects, 2-tailed Spearman's correlation coefficient was used. TT had statistically significant inverse correlation with abdominal circumference at $p<0.01, r=-0.639$ (fig. 1). TT was inversely correlated with overall body fat, measured in kilograms at $p$ $<0.01, r=-0.762$ (fig. 2).

On a biochemical level, significant correlations were found in TT and insulin ( $p<0.01, r=-0.674$ ) (fig. 3 ) and TT with homeostasis model assessment of insulin resistance (HOMA-IR) at $p<0.01, r=-0.623$ (fig. 4).

\section{Discussion}

The study aimed to investigate the relationship between testosterone, body composition, physical function selected biochemical parameters. In our findings, low level of total testosterone correlated with abdominal circumference as a sign of higher change of obesity. These findings agree with (9). They found that abdominal circumference (AC) was inversely correlated with total testosterone $(p=0.006)$ in 159 patients with hypogonadism. Relationship between AC and TT lead to conclusion, that $\mathrm{AC}$ could help to ease the identification of males with low TT levels. Some authors showed relationship between total testosterone and BMI (12), but in our group this correlation was not significant. The correlation between TT and BMI was not that strong and significant as for the abdominal circumference. However, when overall body fat mass was measured in kilograms, we found significant inverse correlation $(p<0.01)$, which also correspond with other studies (1) as one of the primary sign of low TT levels.

On biochemical level, we found significant correlations between total testosterone and insulin ( $p<0.01)$ and HOMA-IR $(p<0.01)$, which can indicate higher incidence of type 2 diabetes and obesity. Similar findings demonstrated also other works with hypogonadal males (13).

The interesting finding of our study was that there was no significant correlation between TT levels and muscle strength, lean mass or physical function in middle age males. These findings are in contradiction to some studies $(1,14)$ which named reduction in lean mass, muscle strength and decreased energy or vitality as one of the primary symptoms of low testosterone levels. Some authors (1) stated that changes in muscle strength in older men correlated with total $(r=0.51 ; P<0.001)$ and free $(r=0.44 ; P<0.001)$ testosterone levels. However, the above $r$ values do not suggest strong correlation of the studied parameters (15), also found that under physiological conditions, testosterone is associated with significant rise in muscle mass providing maximal voluntary strength and decreased fat mass.

In addition, (16) did not find strong correlation between TT and muscle strength and stated that the effects of TT on muscle strength in older men are inconsistent. Some studies in healthy older men have reported improvements in grip strength with higher TT levels. The improvements in strength may be dependent on muscle groups. Muscle groups located on upper body seem to be more responsive on testosterone levels compare to lower body muscles (17). However, there are only limited data available on the beneficial effects of TT on lower limb muscle strength $(16,17)$. 
One of the possible drawback of the study was combination of patients treated with testosterone replacement therapy and untreated males. However, the relationship between parameters studied was very similar in both treated and untreated males, when analysed separately. Therefore, the data were merged for the purpose of the present study.

\section{Conclusions}

The main finding of this study was that testosterone levels had a strong inverse correlation with abdominal circumference and total body fat mass. On metabolic level, strong inverse correlation was also found between TT with insulin and TT with HOMA-IR. However, we did not find statistically significant correlation between total testosterone

\section{REFERENCES}

1. Bhasin S, et al. Testosterone therapy in adult men with androgen deficiency syndromes: an endocrine society clinical practice guideline. J Clin Endocrinol Metab 2006; 91 1995-2010.

2. Bianchi VE, Locatelli V. Testosterone a key factor in gender related metabolic syndrome. Obes Rev 2018; 19: 557-575.

3. Hsu B, Cumming RG, Handelsman DJ. Testosterone, frailty and physical function in older men. Expert Rev Endocrinol Metab 2018: 13: 159-165.

4. Borst SE, Mulligan T. Testosterone replacement therapy for older men. Clinical Interventions in Aging 2007; 2: 561-566.

5. Wang C, et al. REVIEW ISA, ISSAM, EAU, EAA and ASA recommendations: Investigation, treatment and monitoring of late-onset hypogonadism in males. International Journal of Impotence Research 2009; 21: 1-8.

6. Jaworski PED, Ramos A, Nicoleit AR, et al. Importance of abdominal circumference and body mass index values in predicting male hypogonadism; A practical approach. Archives of Endocrinology and Metabolism 2017; 61: 76-80.

7. Corona G, Bianchini S, Sforza A, et al. Hypogonadism as a possible link between metabolic diseases and erectile dysfunction in aging men. Hormones (Athens) 2015; 14: 569-578 8. Santosa S, Jensen MD Effects of male hypogonadism on regional adipose tissue fatty acid storage and lipogenic proteins. PLoS One 2012; 7: e31473.

9. Kelly DM, Jones TH. Testosterone and obesity. Obes Rev 2015; 16: 581-606 levels and lean mass, muscle strength or physical function in middle aged males. These results suggest strong role of testosterone on lipid profile, metabolic syndrome and other similar diseases with altered body composition towards higher proportion of fat tissue. Further studies need to be done for better understanding of the mechanisms how testosterone levels affect overall and regional lean mass, muscle strength, physical function and metabolic parameters in middle age males.

Trial registration: ClinicalTrials.gov: NCT03282682. The study was funded by the Scientific Grant Agency of the Ministry of Education, Science, Research and Sport of the Slovak Republic and of the Slovak Academy of Sciences (VEGA) no. 1/0714/16.

10. Reynolds JM Prediction of one repetition maximum strength from multiple repetition maximum testing and anthropometry. Journal of Strength and Conditioning Research 2006; 20: 584-592.

11. Ebbeling CB, Ward A, Puleo EM, et al. Development of a single-stage submaximal treadmill walking test. Med Sci Sports Exerc 1991; 23: 966-973.

12. Akishita M, Fukai S, Hashimoto M, et al. Association of low testosterone with metabolic syndrome and its components in middle aged Japanese men. Hypertension Res 2010; 33: 587-591

13. Grossmann M et al. Low Testosterone Levels Are Common and Associated with Insulin Resistance in Men with Diabetes. The Journal of Clinical Endocrinology \& Metabolism 2008; 93: 1834-1840.

14. Dandona P, Rosenberg MT. A practical guide to male hypogonadism in the primary care setting. International Journal of Clinical Practice 2010; 64: 682-696.

15. Shamim MO, Khan FMA, Arshad R. Association between serum total testosterone and Body Mass Index in middle aged healthy men. Pak J Med Sci 2015; 31: 355-359.

16. Huhtaniemi I Late-onset hypogonadism: Current concepts and controversies of pathogenesis, diagnosis and treatment. Asian J Androl 2014; 16: 192-202.

17. Hildreth KL, Barry DW, Moreau KL, et al. Effects of testosterone and progressive resistance exercise in healthy, highly functioning older men with low-normal testosterone levels. The Journal of Clinical Endocrinology and Metabolism 2013; 98: 1891-1900. 